Notre Dame Journal of Formal Logic

Volume 47, Number 4, 2006

\title{
Filters on Computable Posets
}

\author{
Steffen Lempp and Carl Mummert
}

\begin{abstract}
We explore the problem of constructing maximal and unbounded filters on computable posets. We obtain both computability results and reverse mathematics results. A maximal filter is one that does not extend to a larger filter. We show that every computable poset has a $\Delta_{2}^{0}$ maximal filter, and there is a computable poset with no $\Pi_{1}^{0}$ or $\Sigma_{1}^{0}$ maximal filter. There is a computable poset on which every maximal filter is Turing complete. We obtain the reverse mathematics result that the principle "every countable poset has a maximal filter" is equivalent to $A C A_{0}$ over $R C A_{0}$. An unbounded filter is a filter which achieves each of its lower bounds in the poset. We show that every computable poset has a $\Sigma_{1}^{0}$ unbounded filter, and there is a computable poset with no $\Pi_{1}^{0}$ unbounded filter. We show that there is a computable poset on which every unbounded filter is Turing complete, and the principle "every countable poset has an unbounded filter" is equivalent to $A C A_{0}$ over $R C A_{0}$. We obtain additional reverse mathematics results related to extending arbitrary filters to unbounded filters and forming the upward closures of subsets of computable posets.
\end{abstract}

\section{Introduction}

In this paper, we study maximal and unbounded filters on computable posets. We obtain computability results and reverse mathematics results regarding the existence of these filters.

We use the following terminology. A poset is a set $P$ with a reflexive, antisymmetric, transitive relation $\preceq$. A poset $\langle P, \preceq\rangle$ is computable if $P$ is a computable subset of $\mathbb{N}$ and $\preceq$ is a computable binary relation on $P$. A filter is a subset $F$ of a poset such that $F$ is upward closed (if $p \in F$ and $p \preceq q$ then $q \in F$ ) and for all $p, q \in F$ there is an $r \in F$ with $r \preceq p$ and $r \preceq q$. The entire poset is thus a filter if every pair of elements is compatible. A filter is maximal if it is not contained in a strictly larger filter. A filter $F$ is unbounded if there is no $p \notin F$ with $p \preceq q$ for all $q \in F$.

Received February 16, 2006; accepted October 16, 2006; printed December 28, 2006 2000 Mathematics Subject Classification: Primary, 03D, 03B30; Secondary, 06

Keywords: computable poset, filter, reverse mathematics

(c) 2006 University of Notre Dame 
In Section 2, we study maximal filters. We show that every computable poset has a $\Delta_{2}^{0}$ maximal filter. This result is optimal: there is a computable poset with no $\Sigma_{1}^{0}$ or $\Pi_{1}^{0}$ maximal filter. There is also a computable poset $P$ such that any maximal filter on $P$ is Turing complete. We obtain a reverse mathematics result: the principle that every countable poset has a maximal filter is equivalent to $A C A_{0}$ over $R C A_{0}$.

In Section 3, we study unbounded filters. We show that every computable poset has a $\Sigma_{1}^{0}$ unbounded filter, and there is a computable poset with no $\Pi_{1}^{0}$ unbounded filter. There is a computable poset $P$ such that every unbounded filter on $P$ is Turing complete. We obtain two reverse mathematics results. The principle that every countable poset has an unbounded filter is equivalent to $A C A_{0}$ over $R C A_{0}$. We define enumerated filters, which are analogous to $\Sigma_{1}^{0}$ filters, and show that the principle "Every sequence of enumerated filters on countable posets extends to a sequence of unbounded enumerated filters" is equivalent to $\mathrm{ACA}_{0}$ over $\mathrm{RCA}_{0}$.

In Section 4, we show that the upward closure of a computable subset of a computable poset may be Turing complete. The principle that every subset of a countable poset has an upward closure is equivalent to $\mathrm{ACA}_{0}$ over $\mathrm{RCA}$.

There has been significant previous research on the computability aspects of linear and partial orders. Downey [1] gives a thorough description of many results. We note that Mummert [2] has shown that there is a computable poset with a computable filter $F$ such that the complete $\Sigma_{1}^{1}$ set is one-one reducible to any extension of $F$ to a maximal filter.

The following results appeared in the Ph.D. thesis of Mummert [3]: Corollaries 2.2 and 4.2 and Theorems 3.1, 3.5, and 3.6. The remaining results are due to both authors.

1.1 Notation We use the following standard notation from computability theory. $W_{e}$ denotes the $\Sigma_{1}^{0}$ set with index $e$ and $W_{e, s}$ denotes the subset of $W_{e}$ which is enumerated in $s$ or fewer steps. $0^{\prime}$ denotes the canonical $\Sigma_{1}^{0}$ complete set. The symbol $\leq_{w t t}$ denotes weak (bounded) truth table reducibility of subsets of $\mathbb{N}$. Readers unfamiliar with these concepts may consult the texts by Rogers [4] or Soare [6].

Reverse mathematics is a program in mathematical logic which classifies theorems based on the set-existence (comprehension) axioms required to prove the theorems. This classification is made using subsystems of second-order arithmetic. In this paper, we use two subsystems of second-order arithmetic: RCA $A_{0}$, which contains $\Delta_{1}^{0}$ comprehension and $\Sigma_{1}^{0}$ induction, and $\mathrm{ACA}_{0}$, which contains arithmetical comprehension and arithmetical induction. A complete definition of these subsystems, along with a complete description of the goals of reverse mathematics, is given by Simpson [5].

\section{Maximal Filters}

A filter on a computable poset is maximal if it is not strictly contained in another filter on the poset.

\section{Theorem 2.1 Every computable poset has a $\Delta_{2}^{0}$ maximal filter.}

Proof Let $P=\left\{p_{0}, p_{1}, \ldots\right\}$ be a computable poset. Begin by forming the oracle $A=\{\langle p, q\rangle \in P \times P \mid \exists r(r \preceq p \wedge r \preceq q)\}$. This oracle is clearly $\Sigma_{1}^{0}$. Now we define a maximal filter $G=\left\{q_{i} \mid i \in \mathbb{N}\right\}$ on $P$ inductively. At stage 0 , let $q_{0}=p_{0}$. At stage $i+1$, we will add two elements $q_{2 i+1}$ and $q_{2 i+2}$ to $G$. First we 
consider element $p_{i+1} \in P$. If $\left\langle p_{i+1}, q_{2 i}\right\rangle \in A$ then we may effectively let $q_{2 i+2}$ be a common extension of $q_{2 i}$ and $p_{i+1}$ and let $q_{2 i+1}=p_{i+1}$. If $\left\langle p_{i+1}, q_{2 i}\right\rangle \notin A$, let $q_{2 i+1}$ and $q_{2 i+2}$ both be $q_{2 i}$. It is not hard to show that $G$ is a maximal filter on $P$. If element $p_{i}$ is added to $G$ then it is added no later than stage $i$; thus $G$ is computable from $A$, so $G$ is $\Delta_{2}^{0}$.

\section{Corollary 2.2 $\quad \mathrm{ACA}_{0}$ proves that every countable poset has a maximal filter.}

Proof The previous proof may be formalized in $\mathrm{ACA}_{0}$ using $\Sigma_{1}^{0}$ comprehension to form $A$.

Theorem 2.3 There is a computable poset $P$ such that $0^{\prime} \leq_{w t t} F$ for any maximal filter $F$ on $P$.

Proof Let $K$ be any $\Sigma_{1}^{0}$ set. For each $k \in \mathbb{N}$, we construct a poset $P_{k}$ inductively. $P_{k}$ begins with two incompatible descending sequences. These sequences will eventually become compatible if $k \in K$; otherwise they will become two incompatible infinite descending sequences. If the sequences do not merge, then exactly one of the two maximal elements of the sequences will appear in any maximal filter, whereas if the sequences do merge then both maximal elements must appear in the unique maximal filter.

Formally, we let $P_{k}=\mathbb{N}$ and define the order $\preceq_{k}$ with the following rules:

$$
\begin{aligned}
2 m \preceq_{k} 2 n & \text { if } n \leq m, \\
2 m+1 \preceq_{k} 2 n+1 & \text { if } n \leq m, \\
2 m \preceq_{k} 2 n+1 & \text { if } n \leq m \text { and } k \in K_{m}, \\
2(m+1)+1 \preceq_{k} 2 n & \text { if } n \leq m \text { and } 2 m+2 \preceq_{k} 2 m+1 .
\end{aligned}
$$

As usual, $K_{m}$ denotes the set of numbers that enter $K$ in no more than $m$ steps of its canonical enumeration. Clearly, $\preceq_{k}$ is a computable partial order. If $k \notin K$ then $2 m \perp 2 n+1$ for all $n, m$, and no maximal filter can contain both 0 and 1 . Otherwise, for all sufficiently large $m, n$ we have $2 m \preceq 2 n+1$ and $2 m+1 \preceq 2 n$, every two elements are compatible, and the unique maximal filter contains 0 and 1.

The construction above is uniform in the sense that we may uniformly compute the order $\preceq_{k}$ from $k$. We may thus form the product poset $P$, which we now describe. The elements of $P$ are finite sequences of natural numbers. We order $P$ by putting $\bar{a} \preceq \bar{b}$ if and only if $|\bar{a}| \geq|\bar{b}|$ and $a_{i} \preceq_{i} b_{i}$ for all $i \leq|\bar{b}|$. Clearly, the order on $P$ is computable, and for any $k \in \mathbb{N}$ and any maximal filter $F$ on $P$ the set $\pi_{k} F=\left\{n \in \mathbb{N} \mid \exists \bar{a} \in F\left(|\bar{a}| \geq k \wedge n=a_{k}\right)\right\}$ is a maximal filter on $P_{k}$.

Note that for each $k$ exactly one of the following options holds.

1. $0 \in \pi_{k} F$ or $1 \in \pi_{k} F$, but not both.

2. $0 \in \pi_{k} F$ and $1 \in \pi_{k} F$.

Beginning with $k=0$ and proceeding inductively, we may effectively determine for each $k$ which of the options holds using no more than 2 queries for each new $k$. For example, we first determine whether 0 or 1 is in $\pi_{0} F$. Assume that $0 \in \pi_{0} F$. Then we can determine whether $1 \in \pi_{1} F$ by asking whether $\langle 0,1\rangle \in F$.

If the first option above holds for $k$ then $k \notin K$. If the second option holds, then $k \in K$. Thus $K \leq_{w t t} G$ for any maximal filter $G$ on $P$. The theorem follows by taking $K=0^{\prime}$. 
Corollary 2.4 The principle that every countable poset has a maximal filter is equivalent to $\mathrm{ACA}_{0}$ over $\mathrm{RCA}_{0}$.

Proof The proof of Theorem 2.3, relativized to an arbitrary oracle, may be formalized in $\mathrm{RCA}_{0}$.

Theorem 2.5 There is a computable poset with no $\Sigma_{1}^{0}$ or $\Pi_{1}^{0}$ maximal filter.

Proof We informally view our poset $P$ as an infinite product of posets $\left\langle P_{e} \mid e \in \mathbb{N}\right\rangle$ and $\left\langle Q_{e} \mid e \in \mathbb{N}\right\rangle$. The construction of $P_{e}$ ensures that $W_{e}$ is not a maximal filter on $P$, and the construction of $Q_{e}$ ensures that the complement of $W_{e}$ is not a maximal filter on $P$.

For each $e$, the poset $P_{e}$ is built as follows. We first construct an infinite descend-

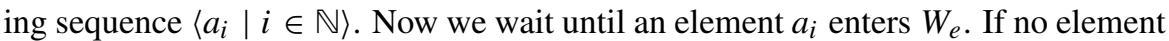
$a_{i}$ enters, then $W_{e}$ is not a maximal filter on $P_{e}$. Whenever an element $a_{i+1}$ enters $W_{e}$, we add an element $b_{i}$ such that $b_{i} \prec a_{i}$ and $b_{i} \perp a_{i+1}$. We then extend $\prec$ so that $b_{i} \prec b_{j}$ if $i>j$ and so that $\prec$ remains transitive. Then we return to waiting. If no element of the form $b_{i}$ is ever added to $W_{e}$, then the infinite descending sequence $\left\langle b_{i}\right\rangle$ generates a filter properly including $W_{e}$. If $W_{e}$ ever includes any element of the form $b_{i}$, we stop adding any new elements of the form $b_{j}$ to the poset. In this case, let $i$ be the largest number such that $b_{i}$ was added to the poset; we know $a_{i+1}$ is in $W_{e}$, and $b_{i} \perp a_{j}$ for all $j \geq i+1$ because we will add no more elements of the form $b_{j}$. Thus $W_{e}$ is not a maximal filter on $P_{e}$.

For each $e$, the poset $Q_{e}$ is built as follows. We construct two infinite descending sequences $\left\langle a_{i}\right\rangle$ and $\left\langle b_{j}\right\rangle$ as in the proof of Theorem 2.3. We wait to see whether $W_{e}$ ever includes $a_{0}$ or $b_{0}$. If it never does, we keep the infinite descending chains incompatible, which means that the complement of $W_{e}$ is not a filter on $Q_{e}$. If $W_{e}$ ever includes $a_{0}$ or $b_{0}$, we cause the two chains to eventually be compatible, which means that the complement of $W_{e}$ is not a maximal filter on $Q_{e}$.

To construct the poset $P$, we view each natural number as a code for a finite sequence of natural numbers, and thus view $P=\mathbb{N}$ as a product poset with one coordinate for each natural number. The order on coordinate $2 i$ of $P$ is similar to the order on poset $P_{i}$, and the order on coordinate $2 i+1$ of $P$ is similar to the order on $Q_{i}$. We watch the effects of each $\Sigma_{1}^{0}$ or $\Pi_{1}^{0}$ subset on the appropriate coordinate of $P$ and use the descriptions of $P_{e}$ and $Q_{e}$ above to shape the order in that coordinate. Because the elements of $P$ are finite sequences and the order relations on $P_{e}$ and $Q_{e}$ are uniformly computable, the order on $P$ will be computable.

\section{Unbounded Filters}

A filter $F$ on a poset $P$ is unbounded if there is no $p \in P \backslash F$ such that $p \preceq q$ for all $q \in F$.

\section{Theorem 3.1 Every computable poset has a $\Sigma_{1}^{0}$ unbounded filter.}

Proof Let $P=\left\langle p_{i} \mid i \in \mathbb{N}\right\rangle$ be a computable poset. We describe an enumeration of an unbounded filter on $P$. At stage 0 , let $q_{0}=p_{0}$. At stage $n+1$, if $p_{i+1} \leq q_{i}$ then let $q_{i+1}=p_{i+1}$; otherwise, let $q_{i+1}=q_{i}$. The upward closure of $\left\{q_{i} \mid i \in \mathbb{N}\right\}$ is easily seen to be a $\Sigma_{1}^{0}$ unbounded filter on $P$. 
Theorem 3.2 There is a computable poset $P$ such that $0^{\prime} \leq_{w t t} F$ for any unbounded filter $F$ on $P$.

Proof We use the computable posets $\left\langle P_{k}, \preceq_{k}\right\rangle$ from the proof of Theorem 2.3. We form a computable poset $P$ whose elements are sequences of the form $\left\langle n, a_{0}, a_{1}, \ldots, a_{n}\right\rangle$ where $n \in \mathbb{N}$ and $a_{k} \in P_{k}$ for each $k \leq n$. We order $P$ by putting $\left\langle n, a_{0}, \ldots, a_{n}\right\rangle \prec\left\langle m, b_{0}, \ldots, b_{m}\right\rangle$ when $n>m$ and $a_{i} \prec_{i} b_{i}$ for each $i \leq m$. Clearly, $P$ is a computable poset; we call this the uniform product of the posets $\left\langle P_{k}\right\rangle$.

We now show that the projection of any unbounded filter on $P$ to coordinate $k+1$ is an unbounded filter on $P_{k}$. Clearly, any unbounded filter on $P$ is generated by an infinite strictly descending sequence $\left\langle r_{i}\right\rangle$ on $P$. Let $p_{i}$ denote the element of $P_{k}$ in coordinate $k+1$ of $r_{i}$ whenever this coordinate exists. The order relation on $P$ ensures that $\left\langle p_{i}\right\rangle$ is an infinite strictly descending sequence on $P_{k}$. Any such sequence determines an unbounded filter on $P_{k}$.

Note that every unbounded filter on $P_{k}$ is maximal; thus we have shown that any unbounded filter on $P$ uniformly computes a maximal filter on $P_{k}$ for each $k$. The remainder of the proof is similar to Theorem 2.3.

We obtain a corollary by formalizing the previous proof in $\mathrm{RCA}_{0}$.

Corollary 3.3 The principle that every countable poset has an unbounded filter is equivalent to $\mathrm{ACA}_{0}$ over $\mathrm{RCA}_{0}$.

The next theorem is proved by applying the uniform product technique of Theorem 3.2 to the sequence of posets $Q_{e}$ in the proof of Theorem 2.5.

Theorem 3.4 There is a computable poset with no $\Pi_{1}^{0}$ unbounded filter.

In $\mathrm{RCA}_{0}$, we define an enumerated filter on a countable poset $\langle P, \preceq\rangle$ to be a function $F$ from $\mathbb{N}$ to $P$ whose range is a filter on $P$. That is, if $p$ is in the range of $F$ and $p \preceq q$ then $q$ is in the range of $F$, and for all $p, q$ in the range of $F$ there is an $r$ in the range of $F$ such that $r \preceq p$ and $r \preceq q$. Note that a filter on $P$ is definable by a $\Sigma_{1}^{0}$ formula if and only if $\mathrm{RCA}_{0}$ is able to form the corresponding enumerated filter. We view an enumerated filter as a code for the set of poset elements it determines, writing $p \in F$ for an enumerated filter $F$ if $p$ is in the range of $F$. We say that an enumerated filter is unbounded if there is no $q \in P$ such that $q \prec p$ whenever $p \in F$. By formalizing the proof of Theorem 3.1, we may prove in $\mathrm{RCA}_{0}$ that every countable poset has an unbounded enumerated filter.

Theorem 3.5 $\quad \mathrm{RCA}_{0}$ proves that every enumerated filter on a countable poset is contained in an unbounded enumerated filter on the poset.

Proof Let $F$ be an enumerated filter on a countable poset $P$. If $F$ is unbounded then we are done. If $F$ is bounded, let $p$ be a lower bound for $F$ and construct an unbounded enumerated filter containing $p$ as in the proof of Theorem 3.1.

The proof of Theorem 3.5 is nonuniform in the sense that the noncomputable choice of whether a given filter is unbounded must be made. The next theorem suggests that no uniform proof of Theorem 3.5 is possible.

Theorem 3.6 The following are equivalent over $\mathrm{RCA}_{0}$.

1. $\mathrm{ACA}_{0}$. 
2. If $\left\langle P_{i} \mid i \in \mathbb{N}\right\rangle$ is a sequence of countable posets and $\left\langle F_{i} \mid i \in \mathbb{N}\right\rangle$ is such that each $F_{i}$ is an enumerated filter on $P_{i}$ then there is a sequence $\left\langle G_{i} \mid i \in \mathbb{N}\right\rangle$ such that $G_{i}$ is an enumerated unbounded filter on $P_{i}$ extending $F_{i}$ for each $i \in \mathbb{N}$.

Proof It is not difficult to prove (2) in $\mathrm{ACA}_{0}$. To prove that (2) implies $A C A_{0}$, we work in $\mathrm{RCA}_{0}$. Let $f: \mathbb{N} \rightarrow \mathbb{N}$ be given; we will show that the range of $f$ exists, which implies $\mathrm{ACA}_{0}$. For each $e \in \mathbb{N}$, form a poset $\left\langle P_{e}, \preceq_{e}\right\rangle$ as follows. $P_{e}$ has an infinite descending sequence $\left\langle a_{i}^{e} \mid i \in \mathbb{N}\right\rangle$ and one additional element $b^{e}$. We let $b^{e} \preceq_{e} a_{i}^{e}$ if there is no $j \leq i$ such that $f(j)=e$. For all $i$ we have $a_{i}^{e} \swarrow_{e} b^{e}$. It is clear that $\preceq_{e}$ is a partial order on $P_{e}$, and the sequence of posets $\left\langle\left\langle P_{e}, \preceq_{e}\right\rangle \mid e \in \mathbb{N}\right\rangle$ may be formed in $\mathrm{RCA}_{0}$.

For each $e \in \mathbb{N}$ let $F_{e}=\left\{a_{i}^{e} \mid i \in \mathbb{N}\right\}$; the sequence $\left\langle F_{e} \mid e \in \mathbb{N}\right\rangle$ may be formed in $\mathrm{RCA}_{0}$ as well. Apply (2) to form a sequence $\left\langle G_{e} \mid e \in \mathbb{N}\right\rangle$ such that each $G_{e}$ is an enumerated filter on $P_{e}$ and $F_{e} \subseteq G_{e}$. Note that $b^{e} \in G_{e}$ if and only if there is no $i \in \mathbb{N}$ such that $f(i)=e$. There is thus a $\Pi_{1}^{0}$ formula which tells whether $e$ is in the range of $f$. Because the range of $f$ has a trivial $\Sigma_{1}^{0}$ definition, we have shown the range of $f$ is definable by a $\Delta_{1}^{0}$ formula, which means that we may form this set in $\mathrm{RCA}_{0}$.

\section{Upward Closures}

The upward closure of a set $F \subseteq P$ is the set $\{q \in P \mid \exists p \in F(p \preceq q)\}$.

Theorem 4.1 There is a computable poset $P$ with a computable linearly ordered subset $F$ such that $0^{\prime}$ is one-one reducible to the upward closure of $F$.

Proof Let $W_{e}$ be a $\Sigma_{1}^{0}$ complete set. We let $P$ have an infinite descending sequence $\left\langle a_{i} \mid i \in \mathbb{N}\right\rangle$ and infinitely many pairwise incompatible elements $\left\{b_{j} \mid j \in \mathbb{N}\right\}$. We put $a_{i} \preceq b_{j}$, if $j \in W_{e, i}$, and $a_{i} \npreceq b_{j}$ otherwise. We always have $b_{j} \npreceq a_{i}$.

The poset just described is computable. Let $A$ denote the computable subset $\left\{a_{i} \mid i \in \mathbb{N}\right\}$. Clearly, $j \in W_{e}$ if and only if $b_{j}$ is in the upward closure of $A$.

Corollary 4.2 The following are equivalent over $\mathrm{RCA}_{0}$.

1. $\mathrm{ACA}_{0}$.

2. Every subset of a countable poset has an upward closure.

3. Every linearly ordered subset of a countable poset has an upward closure.

Proof Because the upward closure of any set $A \subseteq P$ is $\Sigma_{1}^{0}$ definable from $A, \mathrm{ACA}_{0}$ proves (2). Clearly, (2) implies (3). The proof of Theorem 4.1 may be formalized in $\mathrm{RCA}_{0}$ to show that (3) implies $\mathrm{ACA}_{0}$.

\section{References}

[1] Downey, R. G., "Computability theory and linear orderings," pp. 823-976 in Handbook of Recursive Mathematics, Vol. 2, edited by Yu. L. Ershov, vol. 139 of Studies in Logic and the Foundations of Mathematics, North-Holland, Amsterdam, 1998. Zbl 0941.03045. MR 1673590. 480

[2] Mummert, C., "Reverse mathematics of MF spaces," submitted for publication. 480 
[3] Mummert, C., On the Reverse Mathematics of General Topology, Ph.D. thesis, Pennsylvania State University, 2005. 480

[4] Rogers, H., Jr., Theory of Recursive Functions and Effective Computability, 2d edition, The MIT Press, Cambridge, 1987. MR 886890. 480

[5] Simpson, S. G., Subsystems of Second Order Arithmetic, Perspectives in Mathematical Logic, Springer-Verlag, Berlin, 1999. MR 1723993. 480

[6] Soare, R. I., Recursively Enumerable Sets and Degrees, Perspectives in Mathematical Logic, Springer-Verlag, Berlin, 1987. Zbl 0667.03030. Zbl 0623.03042. MR 882921. 480

\section{Acknowledgments}

The first author's research was partially supported by NSF grant DMS-0140120. The second author's research was partially supported by a VIGRE graduate traineeship at the Pennsylvania State University under NSF grant DMS-9810759.

Department of Mathematics

University of Wisconsin

Madison WI 53706-1388

lempp@math.wisc.edu

Department of Mathematics

University of Michigan

Ann Arbor MI 48109-1043

mummertc@umich.edu 